\title{
Virtual Screening of Indonesian Herbal Database as Murine Double Minute-2 (MDM2) Inhibitor
}

\author{
Alexander Victory, Rezi Riadhi Syahdi, Arry Yanuar*
}

\begin{abstract}
Background: Murine Double Minute-2 (MDM2) overexpression causes the p53 deficiency, so the role p53 as a cell regulator does not work in the case of cancer. Methods: In this study, virtual screening of Indonesian herbal database to discover MDM2 inhibitors was carried out. Autodock and Autodock Vina validated with Directory of Useful Decoy-Enhanced (DUD-E). Validation parameters were performed with Enrichment Factor, Receiver Operating Characteristics, and Area Under Curve. Results: The validation with the grid box $70 \times 70 \times 70$ on Autodock resulting AUC value 0.72, while in Autodock Vina 0.43. Autodock Vina did not fulfill the standard value but still used for comparison. Based on the virtual screening result, top ten compounds from Autodock are Nimolicinol, Jacoumaric acid, Isoarborinol, Lantic acid, Diosgenin, Theasaponin E1, Taraxasterol, Leucadenone C, Simiarenol, and Alpha-Amyrin were found to have strong interaction with $\mathrm{MDM} 2$, with binding energy $(\triangle \mathrm{G})$ ranging from -8.83 to $-9.65 \mathrm{kcal} / \mathrm{mol}$. The Autodock Vina screening resulted in the identification of Yuehchukene, Morusin, Cyanidin, Leucadenone C, Roxburghine-B, Ocidentoside, Beta-sitosterol, Curine, Withangulatin, and Jacoumaric acid as potential inhibitors with binding energy $(\triangle \mathrm{G})$ ranging from -8.7 to $-9.4 \mathrm{kcal} / \mathrm{mol}$. Conclusion: Jacoumaric acid and Leucadenone $\mathrm{C}$ were shown to interact with the active site in MDM2 at residues Leu54, Ile61, Met62, and Ile99.
\end{abstract}

Key words: Cancer, Docking, Virtual Screening, Indonesian Herbal, Inhibitor, MDM2.

\section{Alexander Victory, Rezi Riadhi Syahdi, Arry Yanuar ${ }^{*}$}

Faculty of Pharmacy, Universitas Indonesia, 16424, Depok, INDONESIA.

Correspondence

\section{Arry Yanuar}

Faculty of Pharmacy, Universitas Indonesia, 16424, Depok, INDONESIA.

Phone No: +62-21-7270031

E-mail: arry.yanuar@ui.ac.id

\section{History}

- Submission Date: 30-11-2017;

- Review completed: 14-02-2018;

- Accepted Date: 03-05-2018

DOI : 10.5530/pj.2018.6.203

Article Available online

http://www.phcogj.com/v10/i6

\section{Copyright}

(C) 2018 Phcog.Net. This is an openaccess article distributed under the terms of the Creative Commons Attribution 4.0 International license.

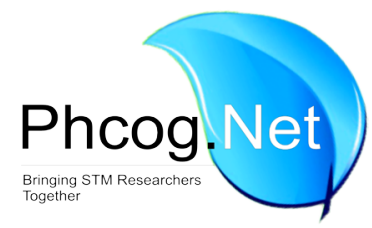

\section{INTRODUCTION}

Chemotherapy treatment has a high level of toxicity so in the treatment of cancer looking for a new approach regarding the development of anticancer that selectively kill cancer cells by triggering apoptosis without damaging healthy cells in the body. ${ }^{1}$ In this research, virtual screening is performed to search for compounds in silico from Indonesian herbal database which has the potential activity to inhibit MDM2 (Murine double minute- 2 homolog) or also known as E3 ubiquitin-protein ligase. Indonesian herbal database is selected as an alternative treatment to exploit the potential of Indonesia's natural wealth, especially in the treatment of cancer and with appropriate development methods, this potential can be utilized as much as possible in the medical world. Virtual screening is used as a complementary method of in vivo and in vitro screening that needs more cost and time.

\section{Indonesian Herbal Database}

Indonesian herbal potential can be found in herbaldb. farmasi.ui.ac.id which is an Indonesian herbal database that already gathers 3810 species with total 6776 compounds. Indonesian herbal database is a small example of Indonesian herbal potential that can use to get the maximum result.

\section{Cell cycle}

The cell cycle is a sequence of events experienced by a cell during its lifetime. The speed of a cell through a cell cycle depends on growth factors, hormones, and chemical factors. The cell cycle is separated into two parts which are interphase and mitosis. ${ }^{2}$

Cancer

Cancer is a disease caused by abnormal growth of body tissue cells that turn into cancer cells. Cancer cells can attack healthy cells around them and can affect everyone in every limb and all age groups. Treatment of cancer by radiotherapy, hormonal therapy, chemotherapy, and biological treatment aimed at increasing the life expectancy of cancer patients. $^{3}$

\section{p53 and Murine Double Minute-2 (MDM2)}

p53 protein level under natural conditions exists at low concentrations in cells, but if stimulated, p53 protein level may increase. This situation is also a stimulation for MDM2 that plays a role in inactivating $\mathrm{p} 53$ by binding to complex or degrading $\mathrm{p} 53$ so that p53 level returns to normal. ${ }^{4}$ MDM2 overexpression causes p53 cell deficiency in many cases of cancer. ${ }^{5}$ The selection of MDM2 as a target in cancer cases by way of inhibition can return the level of p53 to the optimum conditions so that its function as a regulator of cell growth can normally work. ${ }^{6}$ 
MDM2 inhibitors have a purpose of preventing MDM2 binding with p53. MDM2 interaction with p53 occurred on the hydrophobic side of MDM2 and three hydrophobic residues p53 which are Phe19, Trp23, and Leu26. The existence of the MDM2-p53 binding site enables small molecules of inhibitor compounds to inhibit this interaction. ${ }^{7}$ Therapies targeting MDM2 in clinical development include small inhibitor compounds derived from Cis-imidazoline, Spiro-oxindole, Imidazothiazole, Dihydroquinolinone, Piperidines, Piperidone, and Pyrrolidine which have passed the phase 1 clinical trial. ${ }^{8}$ The collected MDM2 inhibitor is summarized as a positive control compound in the study.

\section{METHODS}

\section{Preparation of three-dimensional structure}

MDM2 three-dimensional structure downloaded from RCSB PDB with ID 5LN2. The structure has a resolution of $1.58 \AA$ A. The 1400 ligands used were obtained from an Indonesian herbal database which is accessed from herbaldb.farmasi.ui.ac.id in the form of three dimensions compounds. $^{28}$

\section{Preparation of Three Dimensional Protein Structure}

The search for the macromolecule structure used as the target of the docking is then separated from the residue that can disturb the process so that it will produce suitable docking process. Macromolecular structure preparation starts from searching, selecting, downloading, separating from the unwanted small molecule, and adding of hydrogen atoms and charge. The three-dimensional structure preparation target was done with Protein Data Bank (PDB) and Autodock Tools (ADT).

\section{Docking Optimization}

The optimization of molecular docking is carried out by cocrystal redocking on the target molecule. Docking parameter is Root Mean Square Deviation (RMSD) is lower than $2 \AA$. Inclusion criteria are an MDM2 macromolecule from human-derived, and exclusion criteria were proteins that had a resolution of more than $2.5 \AA$. Macromolecules in this research are MDM2 which has a PDB ID: 5LN2.

\section{Virtual Screening Using Autodock and Autodock Vina}

Screening of compounds from HerbalDB with Autodock and Autodock Vina software. Screening starts with the docking of the compounds in the database on the cocrystal binding site.

\section{Analysis and Visualization of Protein-Ligand Interaction}

The visualization process using PoseView and LigandScout. Visualization is done to see the interaction between ligand and amino acid residues on the macromolecule.

\section{RESULTS AND DISCUSSION}

In docking preparation with Autodock Tools, macromolecule added with hydrogen atoms, merge nonpolar hydrogen, adding Gasteiger charge, then saved in ${ }^{*}$.pdbqt format. The next step is to separate the cocrystal ligand (6ZT) with the 5LN2 macromolecule so can be used for the docking process. The grid box size determination obtained from LigandScout will be used as a reference later for redocking process performed in Autodock. From the result of docking with LigandScout, achieved the size of grid box $60 \times 50 \times 52$ with the center coordinates obtained from the Autodock Tools with the option center on ligand is $\mathrm{x}=-9.442$; $\mathrm{y}=-10.504$; and $\mathrm{z}=0.238$

Negative controls are obtained from the Database of Useful Decoy Enhanced (DUD-E) by accessing dude.docking.org and entering the two-dimensional file of a positive control as shown in Table 1, in the format ${ }^{*}$.smi. The positive control compound files included in DUD-E total is 19 compounds with a total of 1100 decoys of compounds. The positive and negative control files then minimized using OpenBabel by setting the force field with mmff94 and storing a total 1119 compounds in the ${ }^{*}$.pdbqt format which are ready for the validation process with Autoodock and Autodock Vina.

\section{Grid Box Optimization with Autodock}

The optimization step is performed by cocrystal (6ZT) redocking with the macromolecule (5LN2) which have been cleaned from the cocrystal, then looking at the Root Mean Square Deviation (RMSD) value and the binding energy $(\Delta G)$. The data from the redocking result, the lowest RMSD and the highest $\Delta \mathrm{G}$ is used as the parameters in determination of the optimum grid. Grid optimization is done in various grid size with the grid size reference obtained from LigandScout. At first stage of redocking done with Autodock using various grid box size from $30 \mathrm{x} 30 \mathrm{x}$ 30 to $100 \times 100 \times 100$ and repeated ten times (ga_run10) which then will be done the next stage with repetition 100 times (ga_run100).From the redocking result, as shown in the Table 2, the grid box size $70 \mathrm{x} 70 \mathrm{x}$ 70 was chosen as the best grid box size and used for the grid box in the virtual screening process. The value of RMSD is considered can be used if it has a value below $2 \AA .^{29}$

Validation of Virtual Screening Methods with Autodock Enrichment Factor (EF) dan Receiver Operating Characteristics (ROC)

A high value of EF 1\%-2\% signifies a positive control compound that has the high binding energy value in the virtual screening, so it is in the upper section in the order of binding energy value. ${ }^{30}$ Based on the docking result, the best data is on the $70 \times 70 \times 70$ grid box. This result is seen from the emergence of the first positive control data on the first 56 ligands (EF 5\%) so that EF values start to increase in EF 10\% and $\mathrm{EF}$ $20 \%$. The value of EF $1 \%$ has value 0 in all grid sized indicates that positives control do not have a solid interaction compared to ligand decoys. The docking result in Autodock shows that four grid box sizes have a value above the random line which is worth 0.5 . The highest Area Under Curve (AUC) is owned by the $70 \times 70 \times 70$ grid box with AUC 0.7206 , and it is closest to 1 , which is the ideal AUC value. The Area Under Curve Receiver Operating Characteristics (AUROC) $>0.7$ signifies the data is valid to use for validation. ${ }^{31}$

The residues shown are the hydrophobic residues of MDM2 which are Leu54, Leu57, Ile 61, Met62, Tyr67, Val75, Phe86, Phe91, Val93, Ile99, and Ile103 that play a role in the interaction of MDM2 and p53. ${ }^{32}$ The value of EF 1\% in grid box $70 \times 70 \times 70$ in Autodock is 0 . In the Table 3 shown three negative control compounds derived from DUD-E and can be seen the interaction of all the decoys compared with cocrystal 6ZT. All the compounds have highest binding energy but have incomplete interaction to MDM2 macromolecule compared with the cocrystal 6ZT.

Validation of Virtual Screening Methods with Autodock Vina Enrichment Factor (EF) dan Receiver Operating Characteristics (ROC)

Based on the docking result in Autodock Vina, the best data is in the grid box size $15 \times 15 \times 15$ which is equivalent to grid box $40 \times 40 \times 40$ in Autodock. The appearance of the first positive control data on the first 112 ligands (EF 10\%). EF 1\% and EF 5\% value is 0 in all grid size which shows that the positive control does not have a stronger binding energy than the decoys.

The docking result in Autodock Vina obtained one grid box size has an AUC value that passes through the random line. The best AUC value is owned by the grid box $15 \times 15 \times 15$ with AUC value of 0.5053 as shown in the Table 4. The AUC value is not so close to the ideal AUC value and does not pass the value of 0.7 as a parameter of valid data. Although $\mathrm{EF}$ and ROC value does not pass the parameter, virtual screening in Autodock Vina still will be done with the grid box size equivalent of the optimum grid box in Autodock as a comparison. 


\section{Table 1: Positive control compounds}

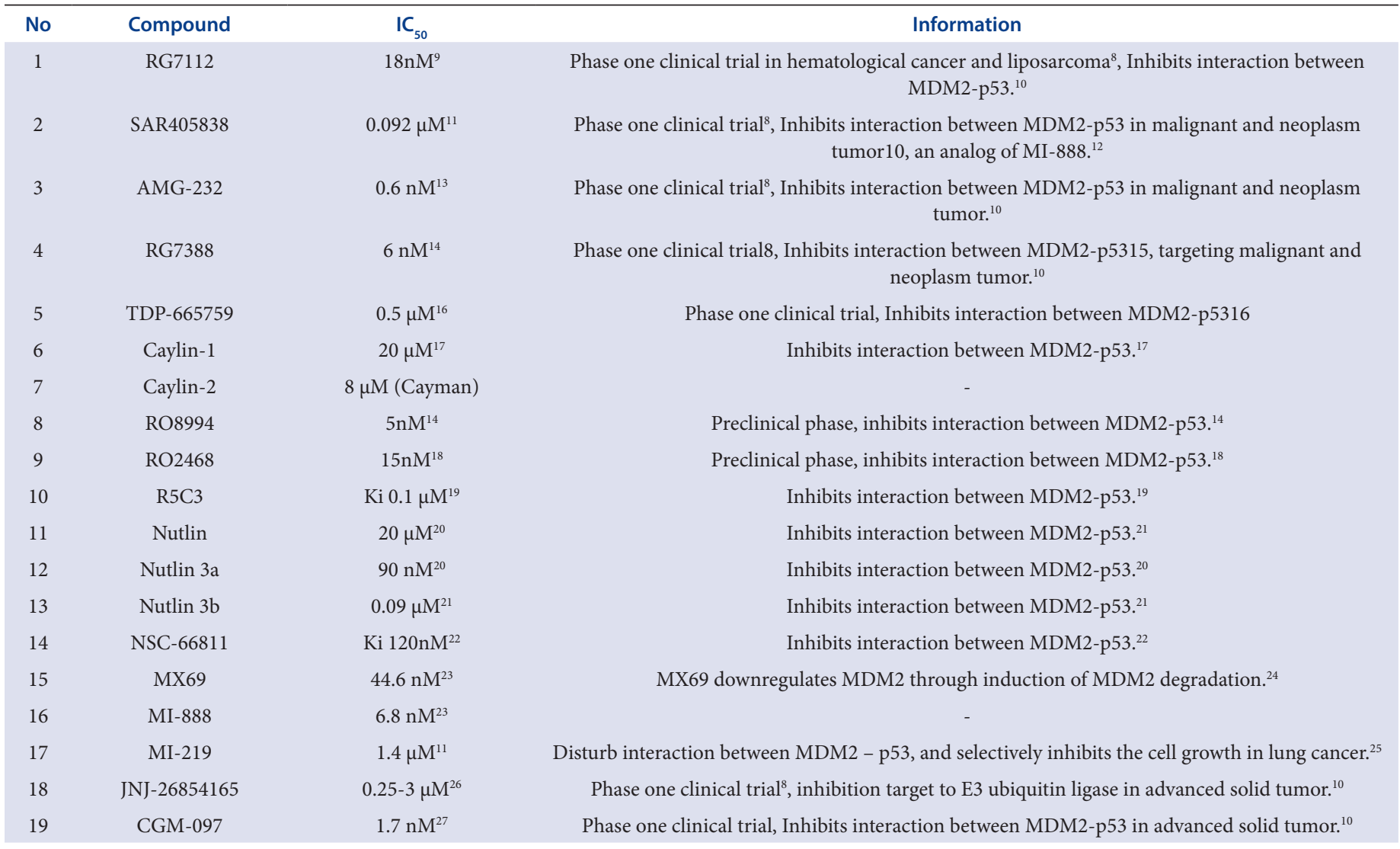

Table 2: Grid box size optimization.

\begin{tabular}{ccc}
\hline Grid box size & Binding energy/ $\Delta \mathrm{G}(\mathrm{kcal} / \mathrm{mol})$ & $\operatorname{RMSD}(\AA ̊)$ \\
\hline $40 \times 40 \times 40$ & -10.30 & 1.47 \\
$70 \times 70 \times 70$ & -10.48 & 1.39 \\
\hline
\end{tabular}

Table 3: Table Interaction between negative control with MDM2 macromolecule.

\begin{tabular}{|c|c|c|c|c|c|c|c|c|c|c|c|}
\hline \multirow[b]{2}{*}{ Compounds } & & \multicolumn{10}{|c|}{ Residues } \\
\hline & & LEU & LEU & ILE & MET & TYR & VAL & PHE & VAL & ILE & ILE \\
\hline & & 54 & 57 & 61 & 62 & 67 & 75 & 86 & 93 & 99 & 103 \\
\hline \multirow[t]{2}{*}{$6 \mathrm{ZT}$} & LS & $\checkmark$ & $\checkmark$ & $\checkmark$ & $\checkmark$ & $\checkmark$ & $\checkmark$ & $\checkmark$ & $\checkmark$ & $\checkmark$ & $\checkmark$ \\
\hline & PV & $\checkmark$ & - & $\checkmark$ & $\checkmark$ & - & - & - & $\checkmark$ & $\checkmark$ & - \\
\hline \multirow[t]{2}{*}{ Decoy 1} & LS & $\checkmark$ & - & $\checkmark$ & - & - & - & - & - & $\checkmark$ & $\checkmark$ \\
\hline & PV & $\checkmark$ & - & $\checkmark$ & - & - & - & - & $\checkmark$ & - & - \\
\hline \multirow[t]{2}{*}{ Decoy 2} & LS & $\checkmark$ & $\checkmark$ & $\checkmark$ & - & - & - & - & - & $\checkmark$ & $\checkmark$ \\
\hline & PV & - & - & - & - & - & - & - & - & - & - \\
\hline \multirow[t]{2}{*}{ Decoy 3} & LS & - & - & - & - & - & - & - & - & - & - \\
\hline & PV & - & - & $\checkmark$ & $\checkmark$ & - & - & - & $\checkmark$ & - & - \\
\hline
\end{tabular}

Table 4: Validation of EF and ROC value with Autodock Vina.

\begin{tabular}{cccccc}
\hline Grid box & EF 1\% & EF 5\% & EF 10\% & EF 20\% & Area Under Curve (AUC) \\
\hline $15 \times 15 \times 15$ & 0 & 0 & 0.52 & 1.31 & 0.5053 \\
$18.75 \times 18.75 \times 18.75$ & 0 & 0 & 0 & 0.52 & 0.4550 \\
$22.5 \times 22.5 \times 22.5$ & 0 & 0 & 0 & 0.52 & 0.4245 \\
$26.25 \times 26.25 \times 26.25$ & 0 & 0 & 0 & 0.78 & 0.4303 \\
\hline
\end{tabular}


Table 5: Top 10 Virtual Screening Result with Autodock and Autodock Vina.

\begin{tabular}{|c|c|c|c|c|}
\hline \multirow[b]{2}{*}{ Rankings } & \multicolumn{2}{|l|}{ Autodock } & \multicolumn{2}{|c|}{ Autodock Vina } \\
\hline & Compound & $\Delta \mathrm{G}(\mathrm{kcal} / \mathrm{mol})$ & Compound & $\Delta \mathrm{G}(\mathrm{kcal} / \mathrm{mol})$ \\
\hline 1 & Nimolicinol & -9.65 & Yuehchukene & -9.4 \\
\hline 3 & Isoarborinol & -9.23 & Cyanidin & -8.8 \\
\hline 4 & Lantic acid & -9.12 & Leucadenone C & -8.8 \\
\hline 6 & Theasaponin E1 & -9.01 & Occidentoseide & -8.7 \\
\hline 7 & Taraxasterol & -8.96 & Beta Sitosterol & -8.7 \\
\hline 8 & Leucadenone C & -8.92 & Curine & -8.7 \\
\hline 9 & Simiarenol & -8.85 & Withangulatin & -8.7 \\
\hline 10 & Alpha-Amyrin & -8.83 & Jacoumaric acid & -8.7 \\
\hline
\end{tabular}

Table 6: Slice of Virtual Screening by Autodock and Autodock Vina.

\begin{tabular}{cccc} 
& & Autodock & Autodock Vina \\
\cline { 3 - 4 } Compound code & Compound & $\Delta \mathrm{G}(\mathrm{kcal} / \mathrm{mol})$ & $\Delta \mathrm{G} \mathrm{(kcal} / \mathrm{mol})$ \\
\hline M00037357 & Jacoumaric acid & -9.24 & -8.7 \\
M00014276 & Leucadenone C & -8.92 & -8.8 \\
\hline
\end{tabular}

Table 7: Interaction of MDM2 amino acid residues with Jacoumaric acid.

\begin{tabular}{|c|c|c|c|c|c|c|c|c|c|c|c|c|c|}
\hline \multicolumn{14}{|c|}{ Residue } \\
\hline & \multirow{2}{*}{ Compound } & & LEU & LEU & ILE & MET & TYR & VAL & PHE & PHE & VAL & ILE & ILE \\
\hline & & & 54 & 57 & 61 & 62 & 67 & 75 & 86 & 91 & 93 & 99 & 103 \\
\hline \multirow{4}{*}{$6 \mathrm{ZT}$} & \multirow{2}{*}{ Autodock } & LS & $\checkmark$ & $\checkmark$ & $\checkmark$ & $\checkmark$ & $\checkmark$ & $\checkmark$ & $\checkmark$ & $\checkmark$ & $\checkmark$ & $\checkmark$ & $\checkmark$ \\
\hline & & PV & $\checkmark$ & & $\checkmark$ & $\checkmark$ & - & - & - & - & $\checkmark$ & $\checkmark$ & . \\
\hline & Autodock & LS & $\checkmark$ & $\checkmark$ & $\checkmark$ & $\checkmark$ & $\checkmark$ & - & $\checkmark$ & $\checkmark$ & - & $\checkmark$ & $\checkmark$ \\
\hline & Vina & PV & $\checkmark$ & - & $\checkmark$ & $\checkmark$ & - & - & - & - & - & $\checkmark$ & - \\
\hline \multirow[t]{2}{*}{ Jacoumaric } & \multirow{2}{*}{ Autodock } & LS & $\checkmark$ & - & $\checkmark$ & $\checkmark$ & - & $\checkmark$ & - & - & $\checkmark$ & $\checkmark$ & - \\
\hline & & PV & $\checkmark$ & - & - & - & - & - & - & - & $\checkmark$ & - & - \\
\hline \multirow[t]{2}{*}{ Acid } & Autodock & LS & $\checkmark$ & - & $\checkmark$ & $\checkmark$ & - & - & - & $\checkmark$ & $\checkmark$ & $\checkmark$ & $\checkmark$ \\
\hline & Vina & PV & $\checkmark$ & - & - & - & - & - & - & - & - & - & - \\
\hline
\end{tabular}

Table 8: Interaction of MDM2 amino acid residues with Leucadenone C

\begin{tabular}{|c|c|c|c|c|c|c|c|c|c|c|c|c|c|}
\hline & & & & & & & & esidue & & & & & \\
\hline & Compound & & LEU & LEU & ILE & MET & TYR & VAL & PHE & PHE & VAL & ILE & ILE \\
\hline & & & 54 & 57 & 61 & 62 & 67 & 75 & 86 & 91 & 93 & 99 & 103 \\
\hline $6 \mathrm{ZT}$ & Autodock & LS & $\checkmark$ & $\checkmark$ & $\checkmark$ & $\checkmark$ & $\checkmark$ & $\checkmark$ & $\checkmark$ & $\checkmark$ & $\checkmark$ & & $\checkmark$ \\
\hline & & PV & $\checkmark$ & . & $\checkmark$ & $\checkmark$ & - & - & - & - & $\checkmark$ & $\checkmark$ & . \\
\hline & Autodock & LS & $\checkmark$ & $\checkmark$ & $\checkmark$ & $\checkmark$ & $\checkmark$ & - & $\checkmark$ & $\checkmark$ & $\checkmark$ & $\checkmark$ & $\checkmark$ \\
\hline & Vina & PV & $\checkmark$ & - & $\checkmark$ & $\checkmark$ & - & - & - & - & - & $\checkmark$ & - \\
\hline Leucadenone $\mathrm{C}$ & Autodock & LS & $\checkmark$ & $\checkmark$ & $\checkmark$ & $\checkmark$ & $\checkmark$ & $\checkmark$ & - & $\checkmark$ & $\checkmark$ & $\checkmark$ & - \\
\hline & 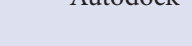 & PV & $\checkmark$ & . & - & $\checkmark$ & - & - & - & - & $\mathrm{D}$ & - & - \\
\hline & Autodock & LS & $\checkmark$ & - & $\checkmark$ & - & - & - & - & $\checkmark$ & $\checkmark$ & $\checkmark$ & $\checkmark$ \\
\hline & Vina & PV & - & - & - & - & - & - & - & - & - & - & - \\
\hline
\end{tabular}




\section{Virtual Screening with Autodock and Autodock Vina}

Table 5 show the result of Autodock and Autodock Vina virtual screening with HerbalDB. Table 6 show the intersection of both virtual screening resulted in two potential compounds as MDM2 inhibitors. Several studies that have been conducted which states the eficacy of both compounds that play a role in inhibiting cancer directly or indirectly Jacoumaric acid comes from the plant Psidium guajava (jambu klutuk) and useful as anticancer. ${ }^{33}$ Leucadenone $\mathrm{C}$ is obtained from a kayu gelang (Melaleuca leucadendron). This plant has four main compounds that efficacious as antioxidants. ${ }^{34}$ Antioxidant-related research as a prevention and treatment of cancer has been done before in an attempt to deal with the impact of cancer on society. ${ }^{35}$

As shown in the Table 7 summary of the amino acid residues that play important role in the MDM2 interaction between cocrystal 6ZT and ligand that appear on the Autodock and Autodock Vina virtual screening processes (Jacoumaric acid). Residues are shown more specific to MDM2 hydrophobic residues which are Leu54, Leu 57, Ile61, Met62, Tyr67, Val75, Phe86, Phe91, Val93, Ile99, and Ile103 that play a role in the interaction of MDM2 and p53. ${ }^{32}$ The cocrystal 6ZT interaction with MDM2 macromolecule is a hydrophobic bond mainly on Leu54, Ile61, Met62, and Ile99. Due to interference on one of those binding sites can interrupt the $\mathrm{p} 53$ and MDM2 bonds. ${ }^{36}$

Jacoumaric acid is obtained from species Psidium guajava, family Myrtaceae which is used as anticancer. ${ }^{33}$ The largest number of interactions that can be seen in visualization results is with Leu54 residue on MDM2. MDM2 interactions with p53 occur on the hydrophobic side of MDM2 and three p53 residues. The presence of the MDM2-p53 binding sites enables small molecules of inhibitor to inhibit this interaction. ${ }^{7}$ Jacoumaric acid has a molecular weight of $618.855 \mathrm{~g} / \mathrm{mol}$ equivalent to 618.855 Dalton. The small molecule inhibitor with the molecular weight between 500-900 Dalton is recommended in the treatment of cancer. ${ }^{37}$ This characteristic supports the potential of Jacoumaric acid as an MDM2 inhibitor.

Leucadenone $\mathrm{C}$ is obtained from kayu gelang plant (Melaleuca leucadendron) family Myrtaceae. Leucadenone A, Leucadenone B, Leucadenone C, Leucadenone D were derived from Melaleuca leucadendron leaf extract. ${ }^{38}$ The Table 8 shows the interaction between cocrystal 6ZT and MDM2 macromolecule mainly a hydrophobic bond on Leu54, Ile61, Met62, and Ile99 residues. The most substantial number of interaction in visualization result is with Leu54 residue on MDM2. Leucadenone $\mathrm{C}$ has a hydrophobic bonding interaction with Leu54 residue. Leucadenone C molecular weight is $540.612 \mathrm{~g} / \mathrm{mol}$ equivalent to 540.612 Dalton. The molecular weight between 500-900 Dalton is recommended in use for cancer treatment. ${ }^{37}$ This characteristic supports the potential of Leucadenone C as an MDM2 inhibitor.

\section{CONCLUSION}

Validation results that match the criteria for positive and negative control using Autodock software with AUC value 0.72, but not for Autodock Vina with AUC 0.43. Virtual screening of the Indonesian herbal database on 5LN2 macromolecule using Autodock and Autodock Vina obtained the top-ten lists which are: nimolicinol, jacoumaric acid, isoarborinol, lantic acid, diosgenin, theasaponin E1, taraxasterol, leucadenone C, simiarenol, alpha-amyrin; and yuehchukene, morusin, cyanidin, leucadenone $\mathrm{C}$, roxburghine $\mathrm{B}$, occidentoside, beta-sitosterol, curine, withangulatin, jacoumaric acid, respectively. The results of virtual screening in Autodock and Autodock Vina are jacoumaric acid and leucadenone $\mathrm{C}$ where the interaction is on Leu54, Ile61, Met62, and Ile99 residues. It is necessary to simulate the molecular dynamics to inspect the bond strength stability of each recommended compound.
Furthermore, in vivo and in vitro tests are required for recommended compounds.

\section{ACKNOWLEDGEMENT}

We are grateful for financial support from PITTA 2017 to AY from Directorate of Research and Community Engagement, Universitas Indonesia.

\section{CONFLICT OF INTEREST}

The authors are declare that there is no conflict of interest.

\section{ABBREVIATIONS}

MDM2: Murine Double Minute-2; PDB: Protein Data Bank; ADT: AutoDock Tools; DUD-E: Database of Useful Decoy - Enhanced; AUC: Area Under Curve; ROC: Receiver Operating Characteristics; AUROC: Area Under Curve Receiver Operating Characteristics; EF: Enrichment Factor; RMSD: Root Mean Square Deviation; RMSF: Root Mean Square Fluctuation; PV: PoseView, LS: LigandScout.

\section{REFERENCES}

1. Sharma G, Kumar N, Singh P, Dubey P, Shankar D, Koch B. p53 dependent apoptosis and cell cycle delay induced by heteroleptic complexes in human cervical cancer cells. Biomed Pharmacother. 2017;88:218-31. Available from: http:// dx.doi.org/10.1016/j.biopha.2017.01.044

2. Corwin EJ. Handbook of Pathophysiology. Third. USA: Lippincott Williams and Wilkins. 2008. 59-84 p.

3. Davies EL. Breast cancer. Medicine (Baltimore) [Internet]. 2016;44(1):42-6

4. Ganguli G, Wasylyk B. p53-independent functions of MDM2. Mol Cancer Res. 2003;1(14):1027-35. Available from: http://www.ncbi.nlm.nih.gov/ pubmed/14707286

5. Gasco M, Shami S, Crook T. The p53 pathway in breast cancer. Breast Cancer Res. 2002:4(2):70-6.

6. Buyukpinarbasili N, Gucin Z, Ersoy YE, Ilbak A, Kadioglu H, Muslumanoglu M. P53 expression and relationship with MDM2 amplification in breast carcinomas. Ann Diagn Pathol. 2016;21:29-34.

7. Wang S, Zhao Y, Bernard D, Aguilar A. Targeting the MDM2-p53 Protein-Protein Interaction for New Cancer Therapeutics. Med Chem 2012;8:57-80.

8. Burgess A, Chia KM, Haupt S, Thomas D, Haupt Y, Lim E. Clinical Overview of MDM2/X-Targeted Therapies. Front Oncol [Internet]. 2016:6:7. Available from: http://www.pubmedcentral.nih.gov/articlerender.fcgi?artid=4728205 and tool=pmcentrez and rendertype=abstract

9. Tovar C, Graves B, Packman K, Filipovic Z, Xia BHM, Tardell C, et al. MDM2 small-molecule antagonist RG7112 activates p53 signaling and regresses human tumors in preclinical cancer models. Cancer Res. 2013;73(8):2587-97.

10. Urso L, Calabrese F, Favaretto A, Conte P, Pasello G. Critical review about MDM2 in cancer: Possible role in malignant mesothelioma and implications for treatment. Crit Rev Oncol Hematol [Internet]. 2016;97:220-

11. Wang S, Sun W, Zhao Y, McEachern D, Meaux I, Barrire C, et al. SAR405838: An optimized inhibitor of MDM2-p53 interaction that induces complete and durable tumor regression. Cancer Res. 2014;74(20):5855-65.

12. Zhao Y, Bernard D, Wang S. Small Molecule Inhibitors of MDM2-p53 and MDMX-p53 Interactions as New Cancer Therapeutics. Biodiscovery [Internet]. 2013;8(8):4

13. Canon J, Osgood T, Olson SH, Saiki AY, Robertson R, Yu D, et al. The MDM2 Inhibitor AMG 232 Demonstrates Robust Antitumor Efficacy and Potentiates the Activity of p53-Inducing Cytotoxic Agents. Mol Cancer Ther [Internet] 2015;14(3):649-58

14. Zhang Z, Ding Q, Liu JJ, Zhang J, Jiang N, Chu XJ, et al. Discovery of potent and selective spiroindolinone MDM2 inhibitor, RO8994, for cancer therapy. Bioorganic Med Chem. 2014;22(15):4001-9.

15. Ribeiro CJA, Rodrigues CMP, Moreira R, Santos MMM. Chemical variations on the p53 reactivation theme. Pharmaceuticals. 2016;9(2):25.

16. Dickens MP, Fitzgerald R, Fischer PM. Small-molecule inhibitors of MDM2 as new anticancer therapeutics. Semin Cancer Biol. 2010;20(1):10-8. Available from: http://dx.doi.org/10.1016/j.semcancer.2009.10.003

17. Verma R, Rigatti MJ, Belinsky GS, Godman CA, Giardina C. DNA damage response to the Mdm2 inhibitor Nutlin-3. Biochem Pharmacol. 2010;79(4):565-74.

18. Zhang Z, Chu XJ, Liu JJ, Ding Q, Zhang J, Bartkovitz D, et al. Discovery of potent and orally active p53-MDM2 inhibitors R05353 and RO2468 for potential clinical development. ACS Med Chem Lett. 2014;5(2):124-7.

19. Zhang R, Mayhood T, Lipari P, Wang Y, Durkin J, Syto R, et al. Fluorescence polarization assay and inhibitor design for MDM2/p53 interaction. Anal Biochem. 
2004;331(1):138-46.

20. Zhao Y, Aguilar A, Bernard D, Wang S. Small-Molecule Inhibitors of the MDM2 - p53 Protein - Protein

21. Vassilev LT. In vivo Activation of the p53 Pathway by Small-Molecule Antagonists of MDM2. Science (80-). 2004;303(5659):844-8.

22. LuY, Nikolovska-Coleska Z, Fang X, Gao W, Shangary S, Qiu S, et al. Discovery of a nanomolar inhibitor of the human murine double min 2 (MDM2)-p53 interaction through an integrated, virtual database screening strategy. J Med Chem. 2006;49(13):3759-62.

23. Zhao Y, Yu S, Sun W, Liu L, Lu J, McEachern D, et al. A potent small-molecule inhibitor of the MDM2-p53 interaction (MI-888) achieved complete and durable tumor regression in mice. J Med Chem. 2013;56(13):5553-61.

24. Gu L, Zhang H, Liu T, Zhou S, DuY, Xiong J, et al. Discovery of Dual Inhibitors of MDM2 and XIAP for Cancer Treatment. Cancer Cell. 2016;30(4):623-36.

25. Zheng MIN, Yang JIE, Xu X, Sebolt JT, Wang S, Sun YI. Efficacy of MDM2 Inhibitor MI-219 Against Lung Cancer Cells Alone or in Combination with MDM2 Knockdown, a XIAP Inhibitor or Etoposide. 2010;30(9):3321-31.

26. Jones R, Gu D, Bjorklund CC, Kuiatse I, Remaley AT, Bashir T, et al. The novel anticancer agent JNJ-26854165 Induces cell death through inhibition of cholesterol transport and degradation of ABCA1s. J Pharmacol Exp Ther. 2013;346(3):381-92

27. Holzer P, Masuya K, Furet P, Kallen J, Valat-Stachyra T, Ferretti S, et al. Discovery of a Dihydroisoquinolinone Derivative (NVP-CGM097): A Highly Potent and Selective MDM2 Inhibitor Undergoing Phase 1 Clinical Trials in p53wt Tumors. J Med Chem. 2015;58(16):6348-58.

28. Yanuar A, Mun'im A, Lagho ABA, Syahdi RR, Rahmat M, Suhartanto $H$. Medicinal Plants Database and Three Dimensional Structure of the Chemi- cal Compounds from Medicinal Plants in Indonesia. Int J Comput Sci. $2011 ; 8(5): 180-3$.

29. Vyas V, Jain A, Jain A, Gupta A. Virtual screening: A fast tool for drug design. Sci Pharm. 2008;76(3):333- 60

30. Zhou N, XuY, Liu X, Wang Y, Peng J, Luo X, et al. Combinatorial pharmacophorebased 3D-QSAR analysis and virtual screening of FGFR1 inhibitors. Int $\mathrm{J} \mathrm{Mol}$ Sci. 2015;16(6):13407-26.

31. Umamaheswari A. E-Pharmacophore BasedVirtual Screening to Identify Agonist for PKA-CÃ and \#142;Â士. Biochem Anal Biochem [Internet]. 2015;4(222):2161 1009

32. Chene P. Inhibition of the p53-MDM2 Interaction: Targeting a Protein-Protein Interface. 2004;2(1):20-8.

33. Sato R, Dang KM, McPherson BG, Brown AC. Anticancer Activity of Guava (Psidium guajava) Extracts. J Complement Integr Med [Internet]. 2010;7(1)

34. Fu L, Xu BT, Xu XR, Qin XS, Gan RY, Li H Bin. Antioxidant capacities and total phenolic contents of 56 wild fruits from South China. Molecules. 2010;15(12):8602-17.

35. Bennett LL, Rojas S, Seefeldt T. Role of Antioxidants in the Prevention of Cancer. J Exp Clin Med. 2012;4(4):215-22.

36. Khoury K, Dömling A. P53 mdm2 inhibitors. Curr Pharm Des [Internet] 2012;18(30):4668-78.

37. Hojjat-Farsangi M. Small-molecule inhibitors of the receptor tyrosine kinases: Promising tools for targeted cancer therapies. Int J Mol Sci. 2014;15(8):13768-801.

38. Goodger JQD, Seneratne SL, Nicolle D, Woodrow IE. Foliar essential oil glands of Eucalyptus subgenus Eucalyptus (Myrtaceae) are a rich source of flavonoids and related non-volatile constituents. PLoS One. 2016;11(3):1-18.

\section{GRAPHICAL ABSTRACT}

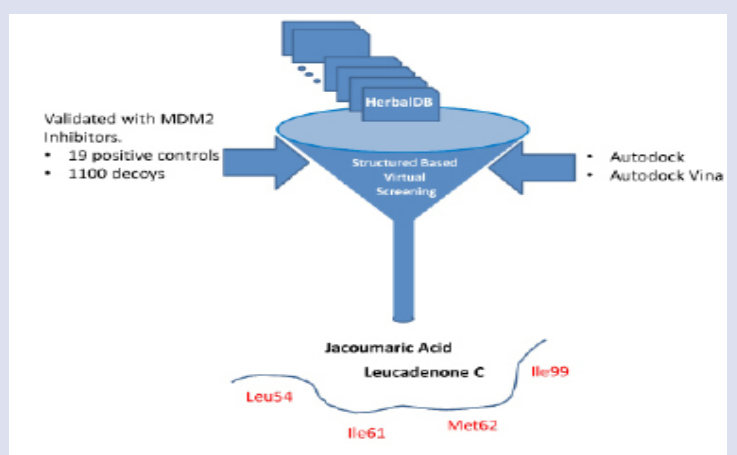

\section{SUMMARY}

- Virtual screening of Indonesian herbal database to discover MDM2 inhibitors was carried out using Autodock and Autodock Vina.

- Top ten virtual screening result compounds from Autodock are Nimolicinol, Jacoumaric acid, Isoarborinol, Lantic acid, Diosgenin, Theasaponin E1, Taraxasterol, Leucadenone C, Simiarenol, and Alpha-Amyrin were found to have strong interaction with $\mathrm{MDM} 2$, with binding energy $(\Delta \mathrm{G})$ ranging from -8.83 to $-9.65 \mathrm{kcal} / \mathrm{mol}$.

- Top ten virtual screening result compounds from Autodock are Yuehchukene, Morusin, Cyanidin, Leucadenone C, Roxburghine-B, Ocidentoside, Beta-sitosterol, Curine, Withangulatin, and Jacoumaric acid as potential inhibitors with binding energy $(\Delta \mathrm{G})$ ranging from -8.7 to $-9.4 \mathrm{kcal} / \mathrm{mol}$.

- Jacoumaric acid and Leucadenone $\mathrm{C}$ were shown to interact with the active site in MDM2 at residues Leu54, lle61, Met62, and lle99.

\section{ABOUT AUTHORS}

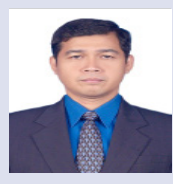

Arry Yanuar: obtained the Bachelor's Degree from Universitas Indonesia in Pharmacy (1990) and Master's degree of Pharmacy from Gadjah Mada University (1997). His PhD in the field of Structural Biology was obtained from Nara Institute of Science and Technology (NAIST), Japan (2006). Currently he works at Faculty of Pharmacy Universitas Indonesia as the head of Laboratory of Biomedical Computation and Drug Design.

Cite this article: Victory A, Syahdi RR, Yanuar A. Virtual Screening of Indonesian Herbal Database as Murine Double Minute2(MDM2) Inhibitor. Pharmacog J. 2018;10(6):1184-9. 